\title{
Adverse events after COVID-19 vaccination: Vector- or spike-induced?
}

\author{
Konstantinos Poulas ${ }^{\dagger}$ \\ 1 University of Patras \\ Funding: The author(s) received no specific funding for this work. \\ Potential competing interests: The author(s) declared that no potential competing interests exist.
}

\begin{abstract}
Efficacious and safe vaccines are needed to contain the COVID-19 pandemic. mRNA and viral vector vaccines are authorized for use in many countries. Cases of thrombosis and/or thrombocytopenia are some of the serious adverse events reported so far. Here we discuss some of these events and try to identify the causative factor of these phenomena.
\end{abstract}

Shultz et al. ${ }^{1}$ reported findings in five patients with venous thrombosis and thrombocytopenia after the administration of the ChAdOx1 vaccine. A close examination of the data presented shows that all patients presented a number of additional postvaccination adverse events, e.g., Patient 1: fever, headaches, visual disturbances; Patient 3: back pain; Patient 5: headache, hemiparesis. Authors describe the phenomenon as a vaccine-induced immune thrombotic thrombocytopenia. Patients had anti-PF4-polyalanine antibodies; however, a control group of serological results by healthy vaccination would have been worth including for comparison purposes.

Greinacher et al. ${ }^{2}$ reported 11 cases of venous thrombosis and thrombocytopenia after the administration of the ChAdOx 1 vaccine. Almost all patients had high levels of anti-PF4-polyanion complex antibodies. A control group of serological results in healthy vaccinated subjects warrants further investigation. One patient reported minor postvaccination symptoms (headache 1 week after vaccination), but information for the other 10 patients was missing. The authors are claiming that this syndrome has been reported only in relation to the ChAdOx1 nCov-19 vaccine.

Scully et al. ${ }^{3}$ reported that 23 patients presented thrombosis and thrombocytopenia after receiving the first dose of ChAdOx1 vaccine with anti-PF4-polyanion complex antibodies. A detailed description of any other postvaccination adverse reactions is not presented, and serological results by healthy vaccinated people are missing. The authors attribute this syndrome to the first dose of ChAdOx1 nCoV-19 vaccine; data about the initiation or not of the second dose in their geographical areas is missing.

Muir et al. ${ }^{4}$ described the case of a patient with extensive thrombosis associated with severe thrombocytopenia and disseminated intravascular coagulation who had received Ad26.COV2. S vaccine. They suggested that rare syndromes, similar to those described previously, ${ }^{1,2,3}$ could be related to adenoviral vector vaccines.

Sadoff et al. ${ }^{5}$, in their attempt to describe the adverse events reported after the administration of Ad26.COV2. vaccine, 
explains that the vectors and spike protein inserts used in the ChAdOx1 vaccine and Ad26.COV2. S vaccine are substantially different. However, although the vectors are totally different, the same does not occur with the spike protein. The part of the spike protein that is ultimately expressed in both vaccines is practically identical. A close examination of data presented by Baden et al. ${ }^{6}$ shows that two deaths occurred after the administration of the mRNA-1273 vaccine in the study group: one from cardiopulmonary arrest and one by suicide. Similarly, Polack et al. ${ }^{7}$ reported two deaths after the administration of the BNT162b2 mRNA vaccine, one from arteriosclerosis and one from cardiac arrest. These deaths are described as unrelated to mRNA vaccines. However, more data are required for three of the four deaths in the study groups. A similar case of postvaccination death after the administration of an mRNA vaccine has been reported but not published ${ }^{8}$ A A 56-year-old MD died after a hemorrhagic stroke due to lack of platelets 15 days after the administration of the BNT162b2 mRNA vaccine.

Lee et al. ${ }^{9}$ also reported cases of apparent secondary immune thrombocytopenia after SARS-CoV-2 vaccination with both the BNT162b2 and mRNA-1273 vaccines. Konstantinidis et al. ${ }^{10}$ presented two cases of smell impairment (hyposmia) after the second dose of the BNT162b2 vaccine. Interestingly, Patient 1 had previous COVID-19 infection with hyposmia without the need for hospitalization.

Any systematic reaction, severe or not, after any nCOV-19 vaccine can be attributed either to the viral vector or to the spike glycoprotein. By combining all the above published data, we propose the hypothesis that spike glycoprotein is the molecule responsible for any adverse effect that is not directly attributed to the expected immune response. It seems that a "Post COVID-19 Vaccination Syndrome (PCovVS)" is developed after the administration of the spike glycoprotein in the human body and should be carefully investigated.

In silico modeling studies suggest that an epitope of the SARS-CoV-2 spike protein adjacent to the receptor-binding domain may interact with alpha7 nicotinic acetylcholine receptors (nAChRs). ${ }^{11}$ These receptors are widely present in different cells, including T lymphocytes and macrophages, and have a pivotal role as part of the cholinergic antiinflammatory pathway. The potential interaction of Spike with alpha7 nAChRs in macrophages was recently described. ${ }^{12}$ Dysregulation of this system could explain many of the clinical manifestations of COVID-19, could be linked to both systemic effects and skin lesions and may play a role in the pathophysiology of severe COVID-19, in which immune dysfunction and hyperinflammatory response appear to be implicated. Since this hypothesis is based on the interaction between Spike and alpha7 nAChRs, it could be applicable to adverse effects related to vaccination (which results in spike production), and this needs to be further explored.

\section{References}

1. Schultz NH, Sorvoll IH, Michelsen AE et al. N Engl J Med. 2021 DOI: 10.1056/NEJMoa2104882

2. Greinacher A, Thiele T, Warkentin TE et al. Thrombotic thrombocytopenia after ChAdOx1 nCov-19 Vaccination. N Engl J Med. 2021 DOI: 10.1056/NEJMoa2104840

3. Scully M, Singh D, Lown R et al. Pathologic antibodies to platelet factor 4 after ChAdOx1 nCoV-19 Vaccination. N Engl J Med. 2021 DOI: 10.1056/NEJMoa2105385

4. Muir KL, Kallam A, Koepsel SA et al. Thrombotic Thrombocytopenia after Ad26.COV2.S Vaccination. N Engl J Med. 
DOI: 10.1056/NEJMc2105869

5. Sadoff J, Davis K, Douoguih M. Thrombotic thrombocytopenia after Ad26.COV2.S vaccination - Response from the manufacturer. N Engl J Med. 2021 DOI: 10.156/NEJMc2106075

6. Baden LR, El Sahly HM, Essink B, et al. Efficacy and Safety of the mRNA-1273 SARS-CoV-2 Vaccine. N Engl J Med. 2021 Feb 4;384(5):403-416. doi: 10.1056/NEJMoa2035389.

7. Polack FP, Thomas SJ, Kitchin N et al. Safety and efficacy of the BNT162b2 mRNA Covid-19 vaccine. N Engl J Med. N Engl J Med 2020; 383:2603-2615

DOI: 10.1056/NEJMoa2034577

8. https://www.nytimes.com/2021/01/12/health/covid-vaccine-death.html

9. Lee EJ, Cines DB, Gernsheimer T et al. Thrombocytopenia following Pfizer and Moderna SARS-CoV-2 vaccination. Am J Hematol. 2021: 1-4.

10. Konstantinidis I, Tsakiropoulou E, Hahner A et al. Olfactory dysfunction after Covid-19 vaccination. International Forum of Allergy \& Rhinology. 2021 In press.

11. Farsalinos K, Eliopoulos E, Leonidas DD, Papadopoulos GE, Tzartos S, Poulas K. Nicotinic Cholinergic System and COVID-19: In Silico Identification of an Interaction between SARS-CoV-2 and Nicotinic Receptors with Potential Therapeutic Targeting Implications. Int J Mol Sci. 2020 Aug 13;21(16):5807. doi: 10.3390/ijms21165807.

12. Tanmay S, Labrou D, Farsalinos K, Poulas K. Is SARS-CoV-2 Spike glycoprotein impairing macrophage function via $\alpha 7$-nicotinic acetylcholine receptors?, Food and Chemical Toxicology. 2021. Volume 152. https://doi.org/10.1016/j.fct.2021.112184. 